Memorias del VII Encuentro Nacional de Experiencias en la Enseñanza de la Biología y la Educación Ambiental y II Congreso Nacional de Investigación en la Enseñanza de la Biología

\title{
LOS HONGOS COMO LENGUAJE Y SIGNIFICADO DEL TERRITORIO EN LA CHAGRA
}

\section{THE FUNGUS AS LANGUAGE AND MEANING OF TERRITORY IN THE CHAGRA}

\section{Lorena González Hurtado}

\section{Resumen}

El artículo muestra la investigación que se llevo a cabo en la Institución Educativa Inga ${ }^{2}$ Yachaikury (Caquetá- Colombia) con los estudiantes del ciclo II, que parte de las intenciones personales y académicas, reconociendo aspectos de la enseñanza de la biología en un contexto intercultural, en donde se pretende fortalecer los procesos de lectura y escritura con significado a partir de los hongos encontrados en la Chagra; siendo esta, una posibilidad de reconocimiento de su cultura, de los saberes tradicionales, de las prácticas agrícolas ancestrales y de la renovación de la memoria, a partir del estudio con los hongos. La investigación tiene un enfoque cualitativointerpretativo, utilizando técnicas etnográficas como observaciones participantes, conversaciones con los mayores, recorridos territoriales, prácticas en la chagra y producción de textos; en la sistematización de la información se proponen categorías de análisis como: conocimientos alrededor de la chagra, conocimiento tradicional y biológico de los hongos, textos y significados de la chagra organizadas en tablas. Se puede concluir que el pretexto del investigador, en este caso los Macrohongos encontrados en la chagra, permite llevar el pensamiento a un punto, donde todos los conocimientos tejidos en el territorio de la comunidad Inga de Caquetá, se pueden expresar en la lectura y escritura con significado.

Palabras clave: Lenguaje y significado, chagra, hongos, conocimiento tradicional, territorio e interculturalidad.

\section{Abstract}

The article shows research that was realized the educational institution Inga Yachaikury (Caquetá - Colombia) with the students of the cycle II, as part of

${ }^{1}$ Estudiante de Licenciatura de Biología. Línea de investigación. Configuración de los conocimientos acerca de lo vivo y la vida Universidad Pedagógica Nacional. Email: loreagonzaleh@gmail.com, dbi.lgonzalez@pedagogica.edu.co

${ }^{2}$ El principal asentamiento del Pueblo Inga es el Valle de Sibundoy, localizado en lo Alto del Rio Putumayo a 2.200 metros sobre el nivel del mar, se encuentran ubicados en varios lugares del territorio nacional, como Aponte- Nariño; Bota Caucana; Medio Putumayo, Bajo Putumayo, Alto Putumayo y Caquetá, en este último ,está ubicado el resguardo de Yurayaco y ocho resguardos más, aunque no se debe desconocer que hay otras familias de esta comunidad en ciudades como: Cali, Barranquilla, Medellín y Bogotá. A nivel histórico por la ola de expansión que sucedió en los años de 1900, en la zona de Putumayo, algunas familias decidieron emigrar al Caquetá defendiendo su territorio, alejándose un poco de las comunidades de origen (Inga P. , 2012). 
Edición Extra-Ordinaria. ISSN 2027-1034 P.p 597 - 606

Memorias del VII Encuentro Nacional de Experiencias en la Enseñanza de la Biología y la Educación Ambiental y II Congreso Nacional de Investigación en la Enseñanza de la Biología

personal and academic intentions, recognizing aspects of the teaching of biology in an intercultural context, where is intended to strengthen the process of reading and writing with meaning from the fungus found on the Chagra; and this like a possibility of recognition of their culture, traditional knowledge, ancestral farming practices and the renewal of the memory, starting with the fungus`s studying.

The research has a methodology qualitative - interpretative, using techniques ethnographic as participating observations, conversations with olders, local tours, practices in the Chagra and production of texts; agree with the information obtained are proposed categories of analysis, such as: knowledge around the chagra, traditional and biological knowledge of fungus, texts and meanings of the chagra organized in tables. It can be concluded that the pretext of the investigator, in this case the Macrofungus found in the chagra, allows thought to a point, where all knowledge woven into the territory of Caquetá Inga community, can be expressed in the reading and writing with meaning.

Key words: language and meaning, chagra, fungus, traditional knowledge, territory and intercultural.

\section{Introducción}

La diversidad social, económica, política, religiosa, científica, etc., que fortalece diferentes aspectos de nuestra existencia, y las diferentes formas que se expresan alrededor de la vida, y dentro de la misma, se toman como interés de investigación lo que se manifiesta en la estrecha relación que hay entre la diversidad cultural y la diversidad biológica, puesto que la variabilidad de los ecosistemas y organismos vivos se interrelaciona con la variabilidad de los modos de vivir, hablar, pensar y comprender el mundo de las comunidades humanas, se trata en fin, de aceptar que "la biodiversidad es también el resultado de procesos culturales"(Sanchez, 2012)

De acuerdo a esto se reconoce a las comunidades indígenas de Colombia, como el lugar, el momento, el sentimiento, la vivencia, la historia; en las cuales se pueden desarrollar proyectos encaminados al reconocimiento del territorio, para este caso el pretexto son los hongos, o como en términos académicos se refiere etnomicología ${ }^{3}$, el cual se enfoca en el aprovechamiento de los recursos para el beneficio de la comunidad y para resaltar los saberes que se tejen alrededor de un aspecto en el cual se referencia lo vivo y la vida; reconociendo todo lo que es y representa su territorio, se promueve una intención de trabajar los microorganismos, en este caso los Macrohongos, que se encuentren en su territorio, en especial en un espacio llamado Chagra, siendo éste, un lugar

${ }^{3}$ En el proceso etnoeducativo, se evita hablar de micología o etnomicología, es decir, no encasillar la investigación a solo un tipo de estudio, sino mas bien mirar desde las diferentes perspectivas o desde diferentes practicas cómo se pueden estudiar los hongos. De todas maneras se entiende por etnomicología, el estudio de las relaciones que existen entre los hombres y los hongos, así como de los saberes tradicionales que de ellos desprenden (Capello, Lopez, \& Sanchez, 2006) 
Edición Extra-Ordinaria. ISSN 2027-1034 P.p 597 - 606

Memorias del VII Encuentro Nacional de Experiencias en la Enseñanza de la Biología y la Educación Ambiental y II Congreso Nacional de Investigación en la Enseñanza de la Biología

característico para la comunidad Inga, donde se reconstruyen sus saberes y se sustentan como territorio y comunidad, dando cuenta, de cómo los hongos encontrados en la chagra de la Institución Educativa Inga Yachaikury, fortalecen los procesos de lectura y escritura con significado a partir del trabajo con los estudiantes del ciclo II $^{4}$.

En términos metodológicos, se tiene en cuenta la ruta metodológica planteada por el pueblo Inga desde su plan de vida y proyecto etnoeducativo, donde se fortalecen desde las ceremonias o rituales de -Ambiwaska-, las mingas, las practicas en la chagra, los recorridos territoriales, conversaciones con los mayores, profesores y estudiantes y el consejo de mayores (Pueblo Inga, 2012). Además se hace uso de técnicas de investigación etnográfica, como la observación participante, y para el registro de la información se utiliza el cuaderno de campo, el registro fotográfico y manual.

A través del análisis de los resultados, se pretende explicar la importancia de los hongos en el territorio del colegio Yachaikury, respondiendo al objetivo central de esta investigación, fortalecer los procesos de lectura y escritura con significado a partir del trabajo con los hongos encontrados en la chagra de la Institución Educativa Inga Yachaikury, la selección de los hongos se da por un interés personal y además porque en la comunidad Inga, los hongos Kayamba-, tienen un significado especial desde el alimento, pero también de los diferentes seres y existencias que viven en el territorio. El reconocimiento de los hongos en campo, se da gracias a los recorridos y prácticas en la Chagra, al conocimiento empírico y tradicional de algunas personas de la comunidad, al registro fotográfico, y a las descripciones que hacen los estudiantes teniendo de acuerdo a su conocimiento.

Gracias a esta ruta metodológica, los objetivos específicos de la investigación dan cuenta de la experiencia vivida en el territorio y del planteamiento del problema los cuales proponen reconocer la chagra, como espacio de recreación de biodiversidad, donde se tejen significados del territorio, caracterizar los hongos desde el conocimiento tradicional y biológico que se encuentran en la chagra de la Institución Educativa Inga Yachaikury y producir textos ${ }^{5}$ que expresen significados de la chagra, construidos a propósito de los hongos y su relación con el territorio.

\footnotetext{
${ }^{4}$ Los sujetos con quienes se trabajo específicamente, son estudiantes del ciclo II, el cual equivale a los niveles de tercero, cuarto y quinto de básica primaria; se caracterizan por ser niños entre los 7 y 11 años de edad, en este ciclo se continua con el proceso de fortalecer la oralidad, y además en explorar diferentes lugares del territorio en el cual habitan, permitiendo realizar actividades como pesca, siembra, recolección, identificación de seres y existencias, relación de su pensamiento con el territorio y además la interpretación de algunos fenómenos naturales que ocurren en -Alpa Mama - madre tierra (Pueblo Inga, 2012). La organización escolar en la institución Inga Yachaikury, es por ciclos, donde cada uno responde a las necesidades de la comunidad escolar y además a la intención del proyecto etnoeducativo.

${ }^{5}$ Texto, viene de la palabra en latín texere, es decir, entrelazar, tejer, para este caso ideas y pensamiento (Pueblo Inga, 2012)
} 
Edición Extra-Ordinaria. ISSN 2027-1034 P. p 597-606

Memorias del VII Encuentro Nacional de Experiencias en la Enseñanza de la Biología y la Educación Ambiental y II Congreso Nacional de Investigación en la Enseñanza de la Biología

La investigación que se desarrollo de acuerdo a la propuesta de práctica pedagógica integral, es importante para identificar desde aspectos interculturales las maneras propias de pensar, pero también de referenciar, la equidad y diversidad de otras culturas, identificando otros saberes aprehendidos y los constructos que teje la comunidad Inga de Yachaikury, como una forma diferente de conocimiento, además por que permiten caminar por los senderos de su territorio, recorriendo los senderos que llevan al éxito de la investigación, resaltando la interacción y el diálogo de saberes que surge entre el saber local y el universal, aprovechando sus fortalezas (Linea de investigación configuración de los conocimientos de lo vivo y la vida, 2011); permitiendo llevar el pensamiento a un punto, donde se contemplen características de forma, de fondo y de interacciones con otras formas de ver el mundo, de sentirlo, de vivirlo, de aprehenderlo y además, de expresarlo.

El desarrollo de la práctica y la estructuración de la misma genera un impacto significativo en la comunidad de la Universidad Pedagógica Nacional, pues como institución de carácter público, y "Educadora de Educadores", es importante que apoye proyectos encaminados al trabajo y reconocimiento de los saberes engendrados en el desarrollo de comunidades indígenas, pues además de aportar a la comunidad académica y científica, puede dar pautas de cómo se entiende el mundo, la vida y lo vivo desde otras perspectivas; además porque se resalta el carácter nacional que debe tener la Universidad, ya que se reconoce su producción, a nivel social y educativo, en la cual significan y se resaltan otros espacios de ser, hacer y saber hacer, maestro, escuela, educación y sociedad como tal.

\section{Diseño metodológico}

Considerar que la investigación promueve la intención de representar de manera significativa la trama de relaciones que se tejen en una sociedad, indica que se debe reflejar el objeto relacionado con el contexto pero evidentemente sin desligarlo del sujeto, siendo esta una triada que esta inquietamente involucrada en la formas de investigar (González, 2012)

Desde la consideraciones éticas para la elección del espacio de desarrollo de la práctica, se tiene en cuenta el acercamiento que se hace gracias a la profesora tutora de éste proyecto, y de las conversaciones con los profesores de la comunidad educativa, pues son ellos los principales interesados y quienes abren las puertas del espacio para tejer pensamiento desde diferentes conocimientos; pero además los intereses personales y académicos para el desarrollo de la misma; es importante resaltar que en términos legales, la comunidad maneja una manera de aceptar los proyectos de investigación por medio de lo establecido en la ley, de acuerdo a la propiedad intelectual y el conocimiento tradicional, utilizando herramientas, como la pre- consulta y consulta previa, donde se tiene en cuenta los aspectos que la comunidad quiere resaltar, pero además los atributos investigativos, científicos, pedagógicos, educativos y culturales que dicho proyecto de práctica puede 
Edición Extra-Ordinaria. ISSN 2027-1034 P.p 597 - 606

Memorias del VII Encuentro Nacional de Experiencias en la Enseñanza de la Biología y la Educación Ambiental y II Congreso Nacional de Investigación en la Enseñanza de la Biología

ofrecer. De todas maneras en relación a esto es importante generar mayor discusión dentro de la comunidad y en la misma academia.

Esta investigación se realiza desde un enfoque cualitativo- interpretativo, donde además se siguen las rutas metodológicas propuestas por el pueblo Inga, desde su propuesta educativa, donde los caminos del saber se abren desde las actividades culturales, cotidianas y ritualizadas como lo es el -Ambiwaska -, remedio, siendo un espacio en el cual los sabedores o Sinchikuna tienen la capacidad de escuchar a la madre tierra (Pueblo Inga, 2012); para que de esta forma, desde la palabra guíen el proyecto de práctica a través del luiaichingapa - consejo de los mayores, siendo éste una característica articuladora en las prácticas agrícolas de la comunidad de Yachaikury, o de cualquier proceso que aporte a resaltar, los caminos del conocimiento lachaiñambikuna- y la renovación de la memoria; las cuales orientan hacia el reconocimiento de la importancia al momento de investigar, de las experiencias vividas, de recorrer el territorio, de conversar, de poner en práctica el consejo de los mayores en la chagra, de tener en cuenta el calendario lunar; siendo estas, estrategias que pueden tener modificaciones de acuerdo a lo que se vaya presentando en el camino.

En síntesis el diseño metodológico se puede describir en cuatro puntos clave: la vivencia en el territorio, el reconocimiento de la chagra, la caracterización de los hongos y la producción textos, ya que permiten que el desenvolvimiento de la temática de práctica, fomente un acercamiento y conexión con la comunidad, donde se resalten los saberes que como licenciada de Biología se han construido, pero también se tengan en cuenta los saberes que allí se construyen, en relación a las prácticas que emergen del territorio, y de la relación intrínseca que tienen con la madre tierra; es decir, que en la medida que estos diálogos se propicien y sean un eje articulador en la práctica, así mismo se construye la ruta para caminar las experiencias y los saberes que de ella sobresalen.

\section{Resultados Y Discusión}

Para la resignificación y reconocimiento de la cultura, la comunidad Inga piensa, en el aspecto de trascender más allá de la palabra escrita alfabéticamente, más allá de lo que en la actualidad se destina para las comunidades con carácter especial en el Territorio Nacional, queriendo ser reconocida como una comunidad que teje sus significados vividos y los que viven, los cuales promueve que se activen los valores culturales para que la nuevas generaciones apropien dichos saberes, que reconfortan de manera significativa lo que son como comunidad y como pueblo que camina por los senderos de su territorio, que se resignifican cada vez que los saberes son puestos en marcha y los relacionan con las realidades que afronta la sociedad, y que además se reconocen como sujetos históricos que hacen parte de un territorio. 
Memorias del VII Encuentro Nacional de Experiencias en la Enseñanza de la Biología y la Educación Ambiental y II Congreso Nacional de Investigación en

Desde este marco de explicación, el Proyecto Etnoeducativo del pueblo Inga, postula como interdisciplinar, los diferentes, encuentros, acercamientos, e intercambios, en donde se retome los hilos del tejido de la cultura, en la cual se proponen lecturas colectivas de territorio desde lugares más próximos hasta los lugares de otros (Proyecto Etnoeducativo, 2012), resaltando el lenguaje y escritura con significado; además que no solo se refiere al desarrollo de habilidades para escribir en Inga y Castellano, sino que también se relaciona con los diferentes tipos de lenguaje, como lo es el arte, los signos, la pintura, el baile, el canto, el mismo tejido, el tallado en madera, etc., esto con el fin de resignificar el pensamiento y la cultura Inga.

En este marco, se desarrollan diversas actividades que apuntan a alcanzar los objetivos propuestos y que además articulan las actividades que la comunidad Inga de Yachaikury realiza, como otros tejidos que aportan a la experiencia, es importante mostrar unas categorías de sistematización que emerjan de lo que se presenta en la experiencia diaria y en algunas necesidades pedagógicas, educativas, didácticas y lúdicas, que el conocimiento tradicional se merece, para el reconocimiento de lo vivo y la vida en los estudiantes del ciclo II.

\begin{tabular}{|c|c|c|}
\hline $\begin{array}{l}\text { CATEGORI } \\
\text { AS }\end{array}$ & $\begin{array}{l}\text { OBJETIVO } \\
\mathrm{S}\end{array}$ & SIGNIFICADO \\
\hline $\begin{array}{l}\text { Conocimien } \\
\text { tos } \\
\text { alrededor } \\
\text { de la chagra }\end{array}$ & $\begin{array}{l}\text { Reconocer } \\
\text { la Chagra } \\
\text { como } \\
\text { espacio de }\end{array}$ & $\begin{array}{l}\text { Se tiene en cuenta las ideas de los estudiantes } \\
\text { del ciclo II, en relación al espacio donde se } \\
\text { siembran los alimentos. } \\
\text { Además de los saberes que se tejen de acuerdo } \\
\text { a las conversaciones con diferentes personas } \\
\text { de la comunidad. }\end{array}$ \\
\hline & $\begin{array}{l}\text { recreación } \\
\text { de la } \\
\text { biodiversida } \\
\text { d, donde se } \\
\text { tejen } \\
\text { significados } \\
\text { del territorio. }\end{array}$ & $\begin{array}{l}\text { La luna se enmarca como un eje articulador } \\
\text { entre los saberes de los mayores y las prácticas } \\
\text { que se llevan a cabo en el territorio, pues se } \\
\text { tiene en cuenta las fases de la luna para } \\
\text { desarrollar aspectos relacionados con la } \\
\text { agricultura ancestral, pero también la relación } \\
\text { de la persona con la chagra. }\end{array}$ \\
\hline $\begin{array}{l}\text { Conocimien } \\
\text { to } \\
\text { tradicional } \\
\text { y biológico } \\
\text { de los } \\
\text { hongos }\end{array}$ & $\begin{array}{l}\text { Caracterizar } \\
\text { desde el } \\
\text { conocimient } \\
\text { o biológico y } \\
\text { tradicional } \\
\text { los hongos } \\
\text { que se } \\
\text { encuentran } \\
\text { en la } \\
\text { Chagra de } \\
\text { la Institución }\end{array}$ & $\begin{array}{l}\text { Desde el conocimiento tradicional, los recorridos } \\
\text { con los mayores, las conversaciones y la } \\
\text { memoria que se guarda entre los orientadores, } \\
\text { sabedores - sinchikuna -, y los mismos } \\
\text { estudiantes. } \\
\text { Desde el conocimiento biológico, lo que el } \\
\text { conocimiento disciplinar puede aportar, las } \\
\text { prácticas en los recorridos, y los saberes que } \\
\text { aporta la academia. Es decir el dialogo } \\
\text { intercultural que se fomenta a la hora de }\end{array}$ \\
\hline
\end{tabular}


Memorias del VII Encuentro Nacional de Experiencias en la Enseñanza de la Biología y la Educación Ambiental y II Congreso Nacional de Investigación en la Enseñanza de La Biología

\begin{tabular}{|c|c|c|}
\hline & $\begin{array}{l}\text { Educativa } \\
\text { Yachaikury }\end{array}$ & interrelacionar los saberes en el territorio. \\
\hline $\begin{array}{l}\text { Lenguaje y } \\
\text { significado } \\
\text { de la } \\
\text { chagra, la } \\
\text { luna y los } \\
\text { hongos }\end{array}$ & $\begin{array}{l}\text { Producir } \\
\text { textos que } \\
\text { expresen } \\
\text { significados } \\
\text { construidos } \\
\text { a propósito } \\
\text { de los } \\
\text { hongos y su } \\
\text { relación con } \\
\text { el territorio. }\end{array}$ & $\begin{array}{l}\text { Entendido como las diferentes construcciones } \\
\text { de los estudiantes, en relación a las prácticas } \\
\text { que se hacen en campo, a lo aprehendido en } \\
\text { las clases con los orientadores, y a los } \\
\text { conocimientos personales del territorio, los } \\
\text { cuales son utilizados para construir significados } \\
\text { desde diferentes tipos de lenguaje, como el } \\
\text { audiovisual, simbólico o ideográfico, entre otros; } \\
\text { permitiendo así tener una visión sistémica de } \\
\text { estos tres puntos que permitan la construcción } \\
\text { de textos que fortalezcan los lazos de } \\
\text { conocimiento en los estudiantes del ciclo II. } \\
\text { Para esta categoría, es importante que el lector } \\
\text { se dé cuenta que en cada actividad, y cualquier } \\
\text { momento de la vivencia los estudiantes están } \\
\text { en constante construcción de significados por } \\
\text { medio de diferentes lenguajes, relacionando } \\
\text { todo lo que representa para ellos, chagra, fases } \\
\text { de la luna y los seres y existencias como los } \\
\text { hongos. }\end{array}$ \\
\hline
\end{tabular}

Tabla 1. Categorías de sistematización, objetivos y su significado

\section{Conclusiones}

$\checkmark$ Se puede establecer que la relación de los estudiantes con el territorio, va más allá de reconocer la chagra como un espacio donde se siembra la biodiversidad, pero también el pensamiento, la idea es que ellos se conviertan en semilla, los cuales buscan a través de la colectividad, un medio adecuado para crecer y fortalecerse desde sus saberes, conocimientos, pensamientos y sentimientos, que reflejen el aprehendizaje a partir de la práctica y de la experiencia vivida.

$\checkmark$ El pretexto utilizado para el desarrollo de la práctica, en un contexto con diferentes miradas de la forma de estar en el mundo y de apropiar el conocimiento, puede ser cualquiera, lo importante es discernir qué es lo verdaderamente pertinente, para establecer puentes de diálogo intercultural entre los diferentes constructos que se tejen en relación a cualquier aspecto que desee explicar el mundo y las formas de asumirlo, para esto es importante que el investigador este con toda la disposición de desaprender para aprender.

$\checkmark$ Los estudiantes del ciclo II, los orientadores, padres de familia, los mayores y los Taitas, desde sus relaciones interpersonales, y con el mismo territorio, permiten elaborar diferentes dinámicas que fortalezcan el proyecto 
Memorias del VII Encuentro Nacional de Experiencias en la Enseñanza de la Biología y la Educación Ambiental y II Congreso Nacional de Investigación en la Enseñanza de La Biología

etnoeducativo de la comunidad Inga y que además generan espacios donde se demuestra una vez más que el trabajo en equipo y la reciprocidad, son las mejores rutas, para que el pensamiento que se construye día a día con la vivencia, sea la excusa perfecta para mantener los hilos que contienen toda la memoria histórica de la comunidad Inga de Yachaikury.

$\checkmark$ La relación que se tejió con la comunidad Inga de Caquetá, promueve que la educación en Yachaikury, este basada en la intención de vivir en armonía con NukanchipaAlpa Mama, reflexionando y asumiendo de manera crítica las relaciones con ella y con otras culturas, construyendo procesos de aprehendizaje; el cual se encamina a la posibilidad de apropiar desentrañando, rebuscando, es decir, ir más allá de lo aparente; desde esta medida en esta escuela no se habla de enseñanza de la biología, sino más bien de la relación que se vive, comparte, y pasa por el corazón y el pensamiento, con todo aquello que se hace llamar madre tierra -Alpa Mama-.

$\checkmark$ La profesión de los maestros está llena de muchos momentos que enriquecen la integralidad del sujeto que enseña y que lo relaciona con el estudiante desde la parte del ser, además que no solo es configurarse como el sujeto que enseña una disciplina, sino que mediante la expresión de su vocación, no solo explica lo que aprende en una carrera profesional, sino que debe preparar para la vida, por ende se debe tener la capacidad de establecer un pensamiento sistémico desde un saber o conocimiento claro.

$\checkmark$ El confort no es el sacrificio de lo material, sino mas bien el sacrifico de la mente, por desligarse de los parámetros que marcan de forma arriesgada desde el momento que somos concebidos; en fin se entiende lo importante que puede ser para el sujeto acercarse al conocimiento de la manera más inquietante que engrandece el espíritu; para este momento es importante establecer que el pensamiento debe partir del todo, y que de esa forma se puede pensar en realizar cosas grandes compartiendo y disfrutando el canto propio que se genera una vez caminemos la vida y las experiencias.

\section{BIBLIOGRAFIA}

- Becerra, W. (1 de Mayo de 2013). Historia, qué es la chagra y su origen. (L. González, Entrevistador)

- Bonilla, E., \& Rodriguez, P. (1998). Más Allá del Dilema de los Métodos. Barcelona: Grupo Editorial Norma.

- Bonilla, V. D. (1968). Siervos de Dios y Amos de Indios. En V. D. Bonilla, Siervos de Dios y Amos de Indios (pág. 317). Bogota: Ediciones Tercer Mundo.

- Buesaquillo, Y. (19 de Febrero de 2013). Historias escritas en relacion a la chagra. Yurayaco, Caquetá. 
Memorias del VII Encuentro Nacional de Experiencias en la Enseñanza de la Biología y la Educación Ambiental y II Congreso Nacional de Investigación en la Enseñanza de la Biología

- Capello, S., Lopez, E., \& Sanchez, V. (2006). Educaciona Ambiental para conocimiento y uso de hongos en una comunidad chontal. Olcuatitán Nacajuca. Tabasco. Horizonte Sanitario , 44- 54.

- Carpenter. P. (1979). microbiologia. Mexico: Interamericana S.A.

- Chalita, E. (19 de Febrero de 2013). Ideas previas en relacion a la Chagra. Yurayaco.

- Dumont, K., Buritica, P., \& Forero, P. (1978). Los Hongos de Colombia. Caldasia.

- Garces. E. (2003). En E. Garces, Morfologia y Clasificacion de los Hongos. Bogotá D.C: Universidad Nacional de Colombia.

- Gobernación de Caquetá, I. G. (2011). Toda Colombia es mi pasión. Recuperado el 23 de Mayo de 2013, de http://www.todacolombia.com/departamentos/caqueta.html

- González, L. (2012).

- Guzman, G. (23 de Noviembre de 1994). La Micologia en america Latina: Pasado, Presente y Futuro. Revista Iberoamericana de Micologia , 81- 85.

- http://www.micomania.rizoazul.com. (s.f.). historia de la micologia. Recuperado el 14 de octubre de 2012, de http://www.micomania.rizoazul.com

- Inga, P. C. (2009). Cartilla Territorio y Cosmovisión, Anexo al Orientador. Colombia: M.E.N.

- Inga, P. (2009). Covenio 455 de 2009, entre el Ministerio de Educacion Nacional y la OZIP (Organización Zonal Indigena del Putumayo). Diagnostico de la situacion educativa del Pueblo Inga, Mocoa.

- Inga, P. (2012). Covenio 455 de 2009, entre el Ministerio de Educacion Nacional y la OZIP (Organización Zonal Indigena del Putumayo). Diagnostico de la situacion educativa del Pueblo Inga, Mocoa.

- Jacanamijoy, B. (16 de Abril de 2013). La Kayamba en el territorio. (L. G. Hurtado, Entrevistador)

- Linea de investigación configuración de los conocimientos de lo vivo y la vida. (2011). Parametros de la Linea de Investigación. Parametros de la Linea de Investigación. Bogotá: Universidad Pedagogica Nacional Departamento de Biologia.

- Lopez, M. (1989). La palabra y la planta. Una aproximacion a la botánica Witoto . Bogotá: Universidad Nacional de Colombia.

- Macas, F. (7 de Marzo de 2013). La importancia de la chagra en la comunidad Inga. (E. Mavisoy, \& F. Ramos, Entrevistadores)

- Martinez, M. e. (11 de Octubre de 2011). Discurso y Aprendizaje, catedra UNESCO para el mejoramiento de la calidad y equidad de la Educacion en America Latina, con base en la Lectura y la Escritura. Recuperado el 2 de Diciembre de 2012, de http://www.unesco-lectura.univalle.edu.co/pdf/

- Modelo Etnoeducativo Inga. (Mayo de 2013).

- Montoya, A., Arias, D., \& Agudelo, M. (2005). Contribucion al conocimiento de los Hongos macromicetos del Resguardo Indigena Nuestra Señora de la 
Memorias del VII Encuentro Nacional de Experiencias en la Enseñanza de la

Biología y la Educación Ambiental y II Congreso Nacional de Investigación en la Enseñanza de la Biología

Candelaria de la Montaña Riosucio- Caldas. Boletin Cientifico- Centro de Museos. Museo de Historia Natural , 19- 30.

- Mutumbajoy, E. (30 de Enero de 2013). Yurayaco.

- Obando, H. (2003). El Lenguaje como Espacio en Blanco para la Significacion al Infinito. En C. U. Dios, Comunicarse: un telar para hilar la resignificación (págs. 22- 29). Bogotá: Codice Ltda.

- Oviedo, S. (19 de Febrero de 2013). Historias escritas por los estudiantes, en relacion a la Chagra. Yurayaco.

- Papalia, D. (1990). Psicologia. Mexico: Mc Graw Hill.

- Proyecto Etnoeducativo. (2012). pueblo Inga , 57. Colombia: Pueblo Inga, M.E.N.

- Pueblo Inga. (2012). Kasami Purinchi Nukanchipa lachaikunawa -Asi caminamos con nuestros saberes- , 57. Colombia: Pueblo Inga, M.E.N.

- Pueblo Inga. (2012). Cartilla Territorio y Cosmovisión, Anexo al Orientador. Colombia: M.E.N.

- Pueblo Inga. (2012). Orientador para el Proyecto Etnoeducativo. Kasami Purinchi Nukanchipa lachaikunawa , 9. Bogotá, Colombia - Caquetá: M.E.N.

- Pueblo Inga. (2009). Texiendo el Pensamiento, anexo al orientador eje Lenguaje y Significados. Colombia: M.E.N.

- Pueblo Inga. (2012). Texiendo el Pensamiento, anexo al orientador eje Lenguaje y Significados. Colombia: M.E.N.

- Salazar, W. (2011). Identificacion de Microhongos aislados de suelos de curtiembres de la cuenca del Rio Tunjuelito . Bogotá, Colombia: Universidad Pedagogica Nacional.

- Sanchez, E. (2012). Colombia Biodiversa: una mirada a nuestros saberes tradicionales. Saberes locales y uso de la biodiversidad en Colombia (pág. 1). Bogotá: Fndacion Ecotrópico Colombia.

- Universidad del Valle de México. (s.f.). Slide Share. Recuperado el 25 de Noviembre de 2012, de http://es.scribd.com/doc/14173444/5b-Siembra-yEstudio-de-Hongos

- Universidad Nacional de Colombia. (s.f.). Sistema de Patrimonio y Museos. Recuperado el 24 de Noviembre de 2012, de http://www.museos.unal.edu.co/sccs/plantilla_museo_3.php?id_subseccion museo $=738 \&$ id_museo $=45$

- Vargas, A. F. (Marzo de 13 de 2013). Los diferentes seres y existencias de la chagra. (L. G. Hurtado, Entrevistador)

- Vasco Palacios, A. M., Suaza, S. C., Castaño Betancur, M., \& Franco Molano, A. E. (2008). Conocimiento etnoecólogico de los hongos entre los indígenas Uitoto, Muinane y andoke de la Amazonia Colombiana. SciELO Brazil, XXXVIII (1), 19.

- Vasco Palacios, A., Franco Molano, A., \& Lopez Quintero, C. (2005). Macromicetes (ascomycota, badidiomycota) de la region del medio Caquetá, departamentos de Caquetá y Amazonas (Colombia). Biota Colombiana, 127- 140. 
Bio-grafía Escritos sobre la Biología y su Enseñanza.

Edición Extra-Ordinaria. ISSN 2027-1034 P. p 597-606

Memorias del VII Encuentro Nacional de Experiencias en la Enseñanza de la

Biología y la Educación Ambiental y II Congreso Nacional de Investigación en la Enseñanza de la Biología

- Zuluaga, C., Buritica, P., \& Marin, M. (2008). Generalidades de los Uredinales (Fungi: Basidiomycota) y de sus relaciones Filogenéticas. Acta Biologica Colombiana , 41- 56. 